\title{
Characterization of Genotypic Mutations and Antiretroviral Resistance among Viremic HIV-Infected Patients in a High HIV Prevalence Area: Treatment Challenge and Transmission Risk ${ }^{*}$
}

\author{
AliAsad Arastu ${ }^{1,2}$, Virginia Kan ${ }^{1,2 \#}$ \\ ${ }^{1}$ Infectious Diseases Section, Veterans Affairs Medical Center, Washington DC, USA; ${ }^{2}$ The George Washington University, Wash- \\ ington DC, USA. \\ Email: "virginia.kan@.va.gov
}

Received June $15^{\text {th }}, 2011$; revised July $4^{\text {th }}, 2011$; accepted July $15^{\text {th }}, 2011$.

\begin{abstract}
There have been few reports evaluating the prevalence of genotypic mutations and antiretroviral resistance among chronic HIV-infected Veterans within the United States. This retrospective cross-sectional study characterizes the rates and changes in HIV genotypic mutations and antiretroviral resistance among viremic patients from 2001 to 2006 at the VA Medical Center located in Washington, DC. The District of Columbia is the metropolitan area with the highest HIV prevalence within the United States. De-identified, linked HIV RNA, genotypic reverse transcriptase (RT) and protease (Pr) mutations and antiretroviral resistance results were assessed for changes during the 6-year period. Aggregated clinic and antiretroviral utilization, and HIV acquisition risk data were evaluated for patients in care during this time. Among 990 viremic samples, the rate of any detected RT or Pr mutation fell from 100\% in 2001 to $95 \%$ in 2006. This was primarily attributable to the $15 \%-20 \%$ decrease seen for RT gene mutations against nucleoside/nucleotide class and non-nucleoside class during this period. Resistance to didanosine, stavudine, zidovudine, nevirapine and efavirenz decreased, and tenofovir resistance increased. Despite stable rates of Pr gene mutations, atazanavir resistance increased by 22\% from 2003 to 2006. Some but not all changes in genotypic mutations and resistance patterns reflected our patients' antiretroviral drug utilization. As sexual contacts (77\%) and injection drug use (22\%) were the leading acquisition risks disclosed by our HIV-infected patients, the high prevalence and changing patterns of HIV genotypic mutations and drug resistance among these patients have had pivotal impacts not only on HIV treatment but potential transmission into our community.
\end{abstract}

Keywords: HIV Viremia, Antiretroviral Resistance, Genotypic Antiretroviral Mutations, Transmission, Nucleoside/ Nucleotide Reverse Transcriptase Inhibitors, Non-Nucleoside Reverse Transcriptase Inhibitors, Protease Inhibitors

\section{Introduction}

Genotypic antiretroviral resistance testing (GART) has

${ }^{*}$ COPYRIGHT STATEMENT: This work has been created in the course of the author's employment by the United States Government and is not subject to copyright.

DISCLOSURE STATEMENT: The views expressed in this article are solely those of the authors and do not reflect the policies of the Department of Veterans Affairs and The George Washington University. FINANCIAL DISCLOSURE: There was no financial support for this study. No competing financial interests exist for the authors. been recommended for primary resistance prior to starting antiretroviral therapy (ART) and for the management of treatment failure in HIV-infected patients [1]. While the prevalence of primary ART resistance has been widely published and reviewed, fewer reports have described such prevalence among ART-treated patients, where patterns and expansion of resistance may have arisen from the selection of variants from patients failing their ART [2]. As part of the HIV Cost and Service 
Utilization Study in the US during 1996-1998, 65\% of 208,900 HIV-infected adults receiving ART had HIV RNA $>500$ copies $/ \mathrm{mL}$, and approximately $76 \%$ of these patients had resistance against more than one drug [3]. Resistance in this study was significantly higher among patients with history of ART, advanced HIV disease, high viremia, and lowest CD4 counts [3]. The prevalence of resistance against one or more drugs has been reported to be between $55 \%$ to $88 \%$ among viremic samples in France [4] and US [5] and between $35 \%$ to $88 \%$ among ART-treated patients in France [2], the UK [6], Thailand [7], and Spain [8].

In the present study, we assessed the prevalence of ART genotypic mutations and resistance among our viremic HIV-infected patients, ART drug utilization, and risks for HIV acquisition during the period 2001-2006 in the District of Columbia, the metropolitan area with the highest HIV prevalence within the United States [9].

\section{Materials and Methods}

The Veterans Affairs Medical Center located in Washington, DC (VAMC-DC) is a tertiary care facility serving Veterans in the greater metropolitan area of the District of Columbia. This study was reviewed in accordance with the ethical standards on human experimentation and with the Helsinki Declaration of 1975 and its revision in 2000 and approved by the Human Studies Subcommittee and Research and Development Committee of the VAMC-DC.

This retrospective, cross-sectional study examined deidentified, linked HIV RNA and GART results during September 2001 through November 2006 at the VAMCDC. HIV RNA was quantified using Versant HIV RNA 3.0 Assay (bDNA) (Siemens Medical Solutions Diagnostics, Tarrytown, NY). GART was assayed using TruGene HIV-1 Genotyping Kit (Siemens Medical Solutions Diagnostics, Tarrytown, NY) using versions 3.0 through 11.0 interpretation rules; mutations in the envelope gene for resistance to entry inhibitors and in the integrase gene for resistance to integrase inhibitors were not assessed by this assay. All tests had been performed for purposes of patient care. GART was performed on clinical samples with detectable viremia including those in the range of 75 1000 copies/mL, as we had previously shown reliable results in our laboratory $[10,11]$.

Aggregated data on the numbers of new HIV diagnoses, patients in HIV care and on ART, and risk factors for HIV acquisition were obtained from our local Clinical Case Registry for HIV. Drug class resistance mutations were based on the IAS-USA December 2010 update [12].

HIV RNA and GART data were analyzed using frequency distribution of mutations and interpreted resis- tance for nucleoside/nucleotide reverse transcriptase inhibitor (NRTI), non-nucleoside reverse transcriptase inhibitor (NNRTI), and protease inhibitor (PI) classes. In addition, $\chi^{2}$ analyses were performed for genotypic mutations and interpreted drug resistance with significance taken at $<0.05$ using STATA (version 8.0, StataCorp LP, College Station, TX).

\section{Results}

Our study evaluated 990 viremic samples with GART results, as summarized in Table 1, with data from our clinic population. Since new HIV diagnoses accounted for $4 \%-9 \%$ of tested patients, most GART were presumably performed for ART failure; however, this could not be confirmed, as we used de-identified data. The proportion of any detected mutations diminished from $100 \%$ in 2001 to $95 \%$ in 2006 . The median rates of mutations to NRTI, NNRTI and PI drug classes were $69 \%$, $47 \%$ and $93 \%$ during this time, respectively. Over the 6-year period, this decrease was attributed to RT mutations, which fell significantly by $13 \%$, while Pr mutations remained relatively stable.

For RT mutations, both NRTI and NNRTI drug classes showed steady decreases in mutations over the study period of $15 \%$ and $20 \%$, respectively. The most prevalent NRTI mutations included M184V/I and thymidine analogue-associated mutations (TAMs) such as M41L, D67N, K70R, L210W, T215V/F, and T219O/E. The mutation rate for $\mathrm{M} 184 \mathrm{~V} / \mathrm{I}$ rose from $38 \%$ to $43 \%$, while rates for TAMs fell from $58 \%$ to $39 \%$. Rates of multi-NRTI resistance due to 69 insertion complex and 151 complex remained relatively stable. The most prevalent NNRTI mutation was $\mathrm{K} 103 \mathrm{~N}$, dropping from $40 \%$ to $26 \%$, while the prevalence of $\mathrm{V} 106 \mathrm{M} / \mathrm{A}$ and $\mathrm{Y} 188 \mathrm{~L} / \mathrm{C} / \mathrm{H}$ remained at $0-2 \%$ and $3 \%-8 \%$, respectively.

Table 2 summarizes the interpreted resistance for ART drugs. Among GART performed on viremic samples, detected resistance to one ART drug ranged from $2 \%$ to $56 \%$ in 2001 and $0.6 \%$ to $43 \%$ in 2006 . Rates of resistance for any NRTI, NNRTI and PI drugs were $2 \%$ $48 \%, 54 \%-56 \%$ and $8 \%-42 \%$ in 2001 , respectively, compared to $9 \%-43 \%, 33 \%-36 \%$ and $0.6 \%-26 \%$ in 2006, respectively. For specific NRTI drugs, resistance remained stable for abacavir and lamivudine/emtricitabine, significantly increased for tenofovir, but declined for didanosine, stavudine and zidovudine. Resistance to efavirenz and nevirapine decreased by at least $20 \%$. Among PI drugs, only atazanavir demonstrated a significant rise in resistance during its years of use from 2003 to 2006, while indinavir and nelfinavir resistance diminished, and amprenavir/fosamprenavir and lopinavir-ritonavir resistance remained without significant 
Table 1. The numbers of clinic patients, patients on antiretroviral therapy (ART), HIV RNA, and genotypic mutations to nucleoside/nucleotide reverse transcriptase inhibitor (NRTI) and non-nucleoside reverse transcriptase inhibitor (NNRTI) and protease inhibitor (PI) drug classes based on annual genotypic antiretroviral resistance tests (GART) are summarized for the study period.

\begin{tabular}{|c|c|c|c|c|c|c|c|}
\hline Year & 2001 & 2002 & 2003 & 2004 & 2005 & 2006 & $\mathrm{P}^{5}$ \\
\hline HIV patients in clinic & 695 & 724 & 746 & 791 & 804 & 809 & \\
\hline patients on ART & 545 & 559 & 570 & 594 & 624 & 642 & 0.41 \\
\hline patients with undetectable viremia & 127 & 171 & 238 & 253 & 311 & 359 & 0.004 \\
\hline median HIV RNA copies $/ \mathrm{mL}^{1}$ & 16,750 & 29,337 & 28,672 & 31,017 & 23,351 & 25,118 & \\
\hline GART done & $50^{2}$ & 162 & 208 & 183 & 177 & $210^{2}$ & \\
\hline GART on patients naive to $\mathrm{ART}^{3}$ & 2 & 11 & 6 & 16 & 10 & 16 & 0.20 \\
\hline \multicolumn{8}{|l|}{ Genotypic mutations $^{4}$} \\
\hline any protease $(\operatorname{Pr})$ mutation & $94 \%$ & $96 \%$ & $92 \%$ & $94 \%$ & $89 \%$ & $92 \%$ & 0.21 \\
\hline any reverse transcriptase (RT) mutation & $78 \%$ & $82 \%$ & $81 \%$ & $68 \%$ & $70 \%$ & $65 \%$ & $<0.001$ \\
\hline to NRTI class & $72 \%$ & $75 \%$ & $74 \%$ & $62 \%$ & $66 \%$ & $57 \%$ & $<0.001$ \\
\hline to NNRTI class & $58 \%$ & $53 \%$ & $57 \%$ & $41 \%$ & $41 \%$ & $38 \%$ & $<0.001$ \\
\hline Any RT or Pr mutation & $100 \%$ & $99 \%$ & $98 \%$ & $97 \%$ & $93 \%$ & $95 \%$ & 0.03 \\
\hline
\end{tabular}

${ }^{1}$ Median HIV RNA reflects the viremic samples linked to GART; ${ }^{2}$ Partial year GART data were available during 9/1-12/31/2001 and 1/1-11/30/2006; ${ }^{3}$ Patients naive to ART was estimated by the numbers of persons not receiving ART from January 1,1981 through December 31 of the year prior to GART; ${ }^{4}$ Percentages are calculated for numbers of gene mutations detected in GART samples; ${ }^{5}$ Two-tailed $\chi^{2}$ analyses were performed with Yates correction.

Table 2. Summary of interpreted resistance to nucleoside/nucleotide reverse transcriptase inhibitor (NRTI), non-nucleoside reverse transcriptase inhibitor (NNRTI), and protease inhibitor (PI) drugs based genotypic antiretroviral resistance tests done, is given for each year of the study.

\begin{tabular}{|c|c|c|c|c|c|c|c|}
\hline Year $^{1}$ & 2001 & 2002 & 2003 & 2004 & 2005 & 2006 & $\mathrm{P}^{3}$ \\
\hline \multicolumn{8}{|l|}{ NRTI resistance } \\
\hline abacavir & $26 \%$ & $31 \%$ & $27 \%$ & $22 \%$ & $21 \%$ & $20 \%$ & 0.44 \\
\hline didanosine & $30 \%$ & $28 \%$ & $23 \%$ & $19 \%$ & $8 \%$ & $14 \%$ & 0.001 \\
\hline lamivudine/emtricitabine & $42 \%$ & $58 \%$ & $49 \%$ & $40 \%$ & $46 \%$ & $43 \%$ & 0.13 \\
\hline stavudine & $32 \%$ & $27 \%$ & $25 \%$ & $27 \%$ & $11 \%$ & $10 \%$ & $<0.001$ \\
\hline tenofovir & $2 \%$ & $1 \%$ & $12 \%$ & $14 \%$ & $15 \%$ & $14 \%$ & $<0.001$ \\
\hline zidovudine & $48 \%$ & $45 \%$ & $37 \%$ & $27 \%$ & $9 \%$ & $9 \%$ & $<0.001$ \\
\hline \multicolumn{8}{|l|}{ NNRTI resistance } \\
\hline efavirenz & $54 \%$ & $48 \%$ & $46 \%$ & $34 \%$ & $33 \%$ & $33 \%$ & 0.004 \\
\hline nevirapine & $56 \%$ & $52 \%$ & $56 \%$ & $40 \%$ & $39 \%$ & $36 \%$ & 0.005 \\
\hline \multicolumn{8}{|l|}{ PI resistance } \\
\hline amprenavir/fosamprenavir & $26 \%$ & $21 \%$ & $17 \%$ & $15 \%$ & $22 \%$ & $22 \%^{2}$ & 0.45 \\
\hline amprenavir/fosamprenavir-ritonavir & & & & & $20 \%$ & $20 \%$ & \\
\hline atazanavir & & & $0 \%$ & $5 \%$ & $22 \%$ & $22 \%$ & $<0.001$ \\
\hline Indinavir & $34 \%$ & $28 \%$ & $19 \%$ & $16 \%$ & $23 \%$ & $24 \%^{2}$ & 0.04 \\
\hline lopinavir/ritonavir & $8 \%$ & $10 \%$ & $12 \%$ & $10 \%$ & $15 \%$ & $15 \%$ & 0.55 \\
\hline nelfinavir & $42 \%$ & $43 \%$ & $33 \%$ & $29 \%$ & $32 \%$ & $26 \%$ & 0.06 \\
\hline tipranavir & & & & & & $0.6 \%$ & \\
\hline
\end{tabular}

${ }^{1}$ Partial year data were available during September 1 through December 31 in 2001 and January 1 through November 30 in $2006 ;{ }^{2}$ Interpreted resistance for both ritonavir-boosted amprenavir/fosamprenamivr and indinavir was $16 \%$ in $2006 ;{ }^{3}$ Two-tailed $\chi^{2}$ analyses were performed with Yates correction. 
change during the study period.

Table 3 summarizes the annual outpatient utilization of individual ART agents during the 6-year period. Ri- tonavir had primarily been used to pharmacologically boost other PI drugs during this time. Tenofovir either singly or in combination formulations had the greatest

Table 3. Patient utilization of antiretroviral agents during 2001 through 2006 is summarized, where data are given as the percent of total HIV patients in care within the Infectious Diseases Clinic for each year.

\begin{tabular}{|c|c|c|c|c|c|c|}
\hline & 2001 & 2002 & 2003 & 2004 & 2005 & 2006 \\
\hline HIV Patients seen in clinic & 695 & 724 & 747 & 790 & 805 & 808 \\
\hline \multicolumn{7}{|l|}{ Nucleoside/Nucleotide RT Inhibitors } \\
\hline abacavir & $4 \%$ & $5 \%$ & $5 \%$ & $5 \%$ & $5 \%$ & $3 \%$ \\
\hline didanosine & $0.6 \%$ & $2 \%$ & $5 \%$ & $7 \%$ & $6 \%$ & $5 \%$ \\
\hline emtricitabine & & & $1 \%$ & $3 \%$ & $3 \%$ & $1 \%$ \\
\hline lamivudine & $21 \%$ & $32 \%$ & $35 \%$ & $32 \%$ & $26 \%$ & $13 \%$ \\
\hline stavudine & $33 \%$ & $28 \%$ & $21 \%$ & $14 \%$ & $12 \%$ & $9 \%$ \\
\hline tenofovir & $2 \%$ & $18 \%$ & $30 \%$ & $33 \%$ & $27 \%$ & $18 \%$ \\
\hline \multicolumn{7}{|l|}{ Non-nucleoside RT Inhibitors } \\
\hline delavirdine & $0.4 \%$ & $0.4 \%$ & $0.4 \%$ & $0.4 \%$ & $0.5 \%$ & $0.2 \%$ \\
\hline efavirenz & $23 \%$ & $32 \%$ & $38 \%$ & $34 \%$ & $32 \%$ & $30 \%$ \\
\hline nevirapine & $7 \%$ & $10 \%$ & $10 \%$ & $8 \%$ & $6 \%$ & $6 \%$ \\
\hline \multicolumn{7}{|l|}{ Protease Inhibitors } \\
\hline atazanavir & & & $4 \%$ & $14 \%$ & $16 \%$ & $18 \%$ \\
\hline darunavir & & & & & & $2 \%$ \\
\hline fosamprenavir & & & & $1 \%$ & $3 \%$ & $2 \%$ \\
\hline indinavir & $11 \%$ & $11 \%$ & $9 \%$ & $5 \%$ & $4 \%$ & $3 \%$ \\
\hline nelfinavir & $14 \%$ & $16 \%$ & $11 \%$ & $8 \%$ & $8 \%$ & $5 \%$ \\
\hline ritonavir & $5 \%$ & $6 \%$ & $6 \%$ & $12 \%$ & $15 \%$ & $18 \%$ \\
\hline saquinavir & & $0.1 \%$ & $0.7 \%$ & $0.9 \%$ & $2 \%$ & $2 \%$ \\
\hline tipranavir & & & & & $1 \%$ & $1 \%$ \\
\hline \multicolumn{7}{|l|}{ Entry Inhibitors } \\
\hline enfuvirtide & & & & $0.3 \%$ & $0.5 \%$ & $2 \%$ \\
\hline \multicolumn{7}{|l|}{ Combination agents } \\
\hline abacavir/lamivudine & & & & $0.5 \%$ & $2 \%$ & $4 \%$ \\
\hline abacavir/lamivudine/zidovudine & $1 \%$ & $3 \%$ & $3 \%$ & $3 \%$ & $4 \%$ & $3 \%$ \\
\hline efavirenz/emtricitabine/tenofovir & & & & & & $7 \%$ \\
\hline emtricitabine/tenofovir & & & & $1 \%$ & $21 \%$ & $31 \%$ \\
\hline lamivudine/zidovudine & $32 \%$ & $38 \%$ & $33 \%$ & $31 \%$ & $29 \%$ & $25 \%$ \\
\hline
\end{tabular}


increase in use from $2 \%$ in 2001 to $56 \%$ in 2006 . Among NNRTI drugs, use of efavirenz alone or in combination formulation rose from $23 \%$ to $37 \%$. Atazanavir and lopinavir/ritonavir use increased during the study period, while indinavir and nelfinavir use declined. In general, a steady increase was seen in the use of formulations which combined medications and/or allowed for a single daily dosing schedule.

Table 4 summarizes the 13 specific risk factors for HIV acquisition disclosed by our HIV-infected patients in care during this study period. Sexual contacts and injection drug use (IDU) accounted for $77 \%$ and $22 \%$ of our patients' acquisition risks, respectively. Continuation of such behavioral risks would allow for potential transmission of resistant HIV from these viremic patients.

\section{Discussion}

There has been one report evaluating rates of HIV genotypic mutations or antiretroviral resistance in the chronically infected Veteran population [13]; there have been none for the District of Columbia, which has the highest rate of persons living with HIV/AIDS in the United States [9]. A prior study done at the VA Medical Center in San Francisco had noted the prevalence of mutations to NRTI, NNRTI, and PI among their patients during
1996-1997 and 1999-2000 to be $30 \%, 14 \%$, and $16 \%$, respectively [13]. In our study at the VAMC-DC during 2001-2006, the prevalence of any genotypic mutations were much higher with median rates of mutations to NRTI, NNRTI and PI classes at $69 \%, 47 \%$ and $93 \%$, respectively.

Among our viremic samples during 2001-2006, the prevalence of any detected mutations fell from $100 \%$ to $95 \%$, a decreasing trend that was seen primarily for NNRTI and NRTI classes of ART. In this period, NNRTI class mutations declined by $20 \%$ with significant drops in efavirenz and nevirapine resistance. During the same period, patients' use of efavirenz (either alone or in combination) increased but nevirapine use remained relatively stable. During 2001-2006, NRTI mutations decreased by $15 \%$ with declining resistance seen for all NRTI drugs except abacavir, lamivudine/emtricitabine and tenofovir. Our patients' use of any tenofovir and lamivudine/emtricitabine increased, and abacavir use remained stable.

During our study period, Pr mutations remained high but relatively unchanged. Resistance was noted to increase only for atazanavir from 2003 to 2006 due to our patients' accumulations of and rising mutation rates for K20R/I, E34Q, M36I, M46I/L, I50L, F53L, I54M, D60E,

Table 4. The specific risk factors for acquisition disclosed by HIV-infected patients in care during 2001 through 2006 are summarized, where the number of individuals (n) and percent of total patients (\%) are given. An individual may have reported one or more risk factors.

\begin{tabular}{|c|c|c|}
\hline & $\mathrm{n}$ & $\%$ \\
\hline Total number of HIV-infected patients in care & 1358 & $100 \%$ \\
\hline \multicolumn{3}{|l|}{ Specific risk factor reported singly or in combination } \\
\hline Sex with Male & 341 & $25 \%$ \\
\hline Sex with Female & 486 & $36 \%$ \\
\hline Injected Nonprescription Drug(s) & 295 & $22 \%$ \\
\hline Received Clotting Factor for Hemophilia/Coagulation Disorder & 0 & $0 \%$ \\
\hline Heterosexual Relations with Bisexual Male & 18 & $1 \%$ \\
\hline Heterosexual Relations with Intravenous Drug User & 166 & $12 \%$ \\
\hline Heterosexual Relations with Person with Hemophilia/Coagulation Disorder & 1 & $0.07 \%$ \\
\hline Heterosexual Relations with Transfusion Recipient with HIV & 1 & $0.07 \%$ \\
\hline Heterosexual Relations with Transplant Recipient with HIV & 1 & $0.07 \%$ \\
\hline Heterosexual Relations with Person with AIDS/HIV infection & 37 & $3 \%$ \\
\hline Received Transfusions Other than Clotting Factor & 16 & $1 \%$ \\
\hline Received Transplant or Artificial Insemination & 0 & $0 \%$ \\
\hline Worked in Healthcare or Laboratory & 12 & $0.8 \%$ \\
\hline
\end{tabular}


A71V/T, G73S, I84V, and L90M [12,14,15]. This increased resistance paralleled our patients' use of atazanavir during the same 4-year period.

Some resistance trends seem discordant with ART utilization for certain drugs such as didanosine, zidovudine, efavirenz and nevirapine, where mutation rates dropped despite stable or higher drug usage rates. Our mutation data were solely from de-identified persons with detectable viremia whose specific ART regimens were not known, while our ART utilization data were from our entire clinic population on ART including those with viral suppression. Importantly, ART-treated patients without detectable viremia (Table 1) increased from $23.3 \%$ in 2001 to $55.9 \%$ in 2006 . Perhaps these persons with viral suppression contributed to the incongruence. Another consideration for the discordance between drug resistance and utilization may be mutations in the connection domain between RT codons 316 and 437 which connect the DNA polymerase and RNase domains. Connection domain mutations were associated with reduced NRTI susceptibility in a study of ART-experienced patients with detectable viremia [16]. As we cannot assess for connection domain mutations in our assay, we do not know if connection domain mutations may have had an impact on the primary RT mutations detected in our GART samples, particularly during the early part of our study period.

A limitation of our de-identified data is the lack of ART history, as historical genotype evaluations rather than a cross-sectional study may give more insight into the prevalence of resistance [17]. Our ART utilization provided only aggregated data for our entire clinic population where a large proportion had viral suppression and thus did not require GART. In addition, our patients' adherence to ART was not evaluated.

The general decrease in transmitted drug resistance over time may be due to wider use of regimens that suppress viral loads or possible selection biases that test for people with fewer mutations [18]. Given the reliability of GART in the viremia range $75-1000$ copies $/ \mathrm{mL}$ in our laboratory $[10,11]$, mutations may be detected earlier among our patients, as their ART resistance emerged.

Decreasing patterns of resistance may likely infer increase in wild type virus with reservoir seeding of more resistant strains. Treating these patients may prove to be difficult, if the true gamut of mutations is not fully captured on testing. More sensitive techniques testing for HIV resistance mutations in peripheral blood mononuclear cells have been used to unmask reservoir viruses [19]; these studies were not pursued in our patients.

Major concerns for viremic patients are contributions to the community viral load and the transmission of re- sistant virus to others. The District of Columbia has had the highest rate of HIV/AIDS in the United States, affecting $3.2 \%$ of persons over age 12 [9]. Our patients' disclosed HIV acquisition risks from sexual contacts (77\%) and IDU (22\%) paralleled those reported by the District of Columbia through 2009, where MSM sexual contact (38.8\%), heterosexual contact (27.2\%) and IDU (16.4\%) were the 3 leading modes of transmission among those living with HIV/AIDS [9]. Behavioral risks among our viremic patients had the potential for transmission of resistant HIV into the community at large, despite the District of Columbia's programs in harm reduction through large scale free condom distribution and needle exchange [9]. Unsafe sexual activities decreased among surveyed patients with ART use [20], ART adherence [20,21], viral suppression [20], optimism about HIV prognosis [22], and prevention interventions [23]. However, suboptimal adherence and current or past history of drug and/or alcohol abuse were among the predictors for virologic failure [24]. In addition, patients reporting IDU with or without opiate substitution treatment had a 2- to 3 -fold higher non-adherence to ART compared with abstinent patients [25]. These persons with reduced adherence to ART may engage in more risk behaviors, thereby allowing further transmission of resistant HIV.

The high prevalence and changing patterns of HIV genotypic mutations and drug resistance among our viremic patients have pivotal impact not only on our ART management but also on potential transmission of resistant HIV into the metropolitan community with the highest HIV prevalence within the United States.

\section{Acknowledgements}

We thank Howard B. Gale, MD, Infectious Diseases Laboratory supervisor, for provision of de-identified genotypic antiretroviral resistance test data and critical review of this manuscript, and Katherine A. Hare, BS, for assistance with manuscript preparation.

\section{REFERENCES}

[1] The HHS Panel on Antiretroviral Guidelines for Adults and Adolescents, "Guidelines for the Use of Antiretroviral Agents in HIV-Infected Adults and Adolescents," 2011. http://aidsinfo.nih.gov/contentfiles/AdultandAdolescentG L.pdf

[2] D. Costagliola, D. Descamps, L. Assoumou, L. MorandJoubert, A. G. Marcelin, V. Brodard, C. Delaugerre, V. Mackiewicz, A. Ruffault, J. Izopet, J. C. Plantier, C. Tamalet, S. Yerly, S. Saidi, F. Brun-Vezinet, B. Masquelier and Agence Nationale de Recherches sur le SIDA et les Hepatites Virales (ANRS) AC11 Resistance Study Group, "Prevalence of HIV-1 Drug Resistance in Treated Patients: A French Nationwide Study," Journal of Acquired 
Immune Deficiency Syndromes, Vol. 46, No. 1, 2007, pp. 12-18.

[3] D. D. Richman, S. C. Morton, T. Wrin, N. Hellmann, S. Berry, M. F. Shapiro and S. A. Bozzette, "The Prevalence of Antiretroviral Drug Resistance in the United States," AIDS, Vol. 18, No. 10, 2004, pp. 1393-1401. doi:10.1097/01.aids.0000131310.52526.c7

[4] C. Tamalet, J. Fantini, C. Tourres and N. Yahi, "Resistance of HIV-1 to Multiple Antiretroviral Drugs in France: A 6-Year Survey (1997-2002) Based on an Analysis of Over 7000 Genotypes," AIDS, Vol. 17, No. 16, 2003, pp. 2383-2388. doi:10.1097/00002030-200311070-00014

[5] R. Kagan, M. Winters, T. Merigan and P. Heseltine, "HIV Type 1 Genotypic Resistance in a Clinical Database Correlates with Antiretroviral Utilization," AIDS Research Human Retroviruses, Vol. 20, No. 1, 2004, pp. 1-9. doi: $10.1089 / 088922204322749440$

[6] P. Scott, E. Arnold, B. Evans, A. Pozniak, G. Moyle, M. Shahmenesh, D. White, J. Shirley, P. Cane and D. Pillay, "Surveillance of HIV Antiretroviral Drug Resistance in Treated Individuals in England: 1998-2000," Journal of Antimicrobial Chemotherapy, Vol. 53, No. 3, 2004, pp. 469-473. doi:10.1093/jac/dkh102

[7] C. Sukasem, V. Churdboonchart, S. Chasombat, S. Kohreanudom, C. Watitpun, W. Piroj, M. Tiensuwan and W. Chantratita, "Surveillance of Genotypic Resistance Mutations in Chronic HIV-1 Treated Individuals after Completion of the National Access to Antiretroviral Program in Thailand," Infection, Vol. 35, No. 2, 2007, pp. 81-88. doi:10.1007/s15010-007-6169-x

[8] O. Gallego, L. Ruiz, A. Vallejo, E. Ferrer, A. Rubio, B. Clotet, M. Leal, V. Soriano and ERASE-3 Group, "Changes in the Rate of Genotypic Resistance to Antiretroviral Drugs in Spain," AIDS, Vol. 15, No. 14, 2001, pp. 18941896. doi:10.1097/00002030-200109280-00025

[9] The Department of Health, "The District of Columbia HIV/AIDS, Hepatitis, STD and TB (HAHSTA) Annual Report 2010," published 15 June 2011, accessed on 22 June 2011.

http://dchealth.dc.gov/doh/frames.asp?doc=/doh/lib/doh/s ervices/administration_offices/hiv_aids/pdf/2010_Annual Report_FINAL.pdf

[10] H. B. Gale, V. L. Kan and R. C. Shinol, "Performance of the Tru-Gene Human Immunodeficiency Virus Type 1 Genotyping Kit and Open-Gene DNA Sequencing System on Clinical Samples Diluted to Aapproximately 100 Copies per Milliliter," Clinical Vaccine Immunolology, Vol. 13, 2006, pp. 235-238. doi:10.1128/CVI.13.2.235-238.2006

[11] V. L. Kan, H. B. Gale and R. C. Shinol, "Response to Waters et al. 'Successful Use of Genotypic Resistance Testing in HIV-1-Infected Individuals with Detectable Viraemia'," AIDS, Vol. 20, No. 15, 2006, pp. 1991-1992. doi:10.1097/01.aids.0000247129.65623.d1

[12] V. A. Johnson, F. Brun-Vézinet, B. Clotet, H. F. Günthard, D. R. Kuritzkes, D. Pillay, J. M. Schapiro and D. D. Richman, "Update of the Drug Resistance Mutations in
HIV-1: December 2010," Topics in HIV Medicine, Vol. 18, 2010, pp. 156-163.

[13] M. Holodniy, E. D. Charlebois, D. R. Bangsberg, A. R. Zolopa, M. Schulte and A. R. Moss, "Prevalence of Antiretroviral Drug Resistance in the HIV-1-Infected Urban Indigent Population in San Francisco: A Representative Study," International Journal of STD and AIDS, Vol. 15, No. 8, 2004, pp. 543-551. doi: $10.1258 / 0956462041558212$

[14] S. Vora S, A. G. Marcelin, H. F. Günthard, P. Flandre, H. H. Hirsch, B. Masquelier, A. Zinkernagel, G. Peytavin, V. Calvez, L. Perrin, S. Yerly and Swiss HIV Cohort Study, "Clinical Validation of Atazanavir/Ritonavir Genotypic Resistance Score in Protease Inhibitor-Experienced Patients," AIDS, Vol. 20, No. 1, 2006, pp. 35-40. doi:10.1258/0956462041558212

[15] M. M. Santoro, A. Bertoli, P. Lorenzini, F. CeccheriniSilberstein, N. Gianotti, C. Mussini, C. Torti, G. Di Perri, G. Barbarini, T. Bini, S. Melzi, P. Caramello, R. Maserati, P. Narciso, V. Micheli, A. Antinori, C. F. Perno and CARe Study Group, "Two Different Patterns of Mutations Are Involved in the Genotypic Resistance Scores of Atazanavir Boosted Versus Unboosted by Ritonavir in Multiple Failing Patients," Infection, Vol. 37, No. 3, 2009, pp. 233-243.

[16] B. Dau, D. Ayers, J. Singer, P. R. Harrigan, S. Brown, T. Kyriakides, D. W. Cameron, B. Angus and M. Holodniy, "Connection Domain Mutations in Treatment-Experienced Patients in the OPTIMA Trial," Journal of Acquired Immune Deficiency Syndromes, Vol. 54, No. 2, 2010, pp. 160-166.

[17] P. R. Harrigan, B. Wynhoven, Z. L. Brumme, C. J. Brumme, B. Sattha, J. C. Major, R. de la Rosa and J. S. Montaner, "HIV-1 Drug Resistance: Degree of Underestimation by a Cross-Sectional Versus a Longitudinal Testing Approach," Journal of Infectious Diseases, Vol. 191, No. 8, 2005, pp. 1325-1330. doi:10.1086/428852

[18] UK Collaborative Group on HIV Drug Resistance, UK Collaborative HIV Cohort Study, and UK Register of HIV Seroconverters, "Evidence of a Decline in Transmitted HIV-1 Drug Resistance in the United Kingdom," AIDS, Vol. 21, No. 8, 2007, pp. 1035-1039. doi:10.1097/QAD.0b013e3280b07761

[19] O. Turriziani, M. Bucci, A. Stano, C. Scagnolari, F. Bellomi, C. Fimiani, I. Mezzaroma, G. D’Ettorre, A. Brogi, V. Vullo and G. Antonelli, "Genotypic Resistance of Archived and Circulating Viral Stains in the Blood of Treated HIV-Infected Individuals," Journal of Acquired Immune Deficiency Syndromes, Vol. 44, 2007, pp. 518524. doi:10.1097/QAI.0b013e3180315515

[20] C. Diamond, J. L. Richardson, J. Milam, S. Stoyanoff, J. A. McCutchan, C. Kemper, R. A. Larsen, H. Hollander, P. Weismuller, R. Bolan and California Collaborative Trials Group, "Use of and Adherence to Antiretroviral Therapy Is Associated with Decreased Sexual Risk Behavior in HIV Clinic Patients," Journal of Acquired Immune Deficiency Syndromes, Vol. 39, 2005, pp. 211-218. 
[21] S. C. Kalichman and D. Rompa, "HIV Treatment Adherence and Unprotected Sex Practices in People Receiving Antiretroviral Therapy," Sexually Transmitted Infection, Vol. 79, No. 1, 2003, pp. 59-61. doi:10.1136/sti.79.1.59

[22] W. C. Holmes and J. L. Pace, "HIV-Seropositive Individuals' Optimistic Beliefs about Prognosis and Relation to Medication and Safe Sex Adherence," Journal of General Internal Medicine, Vol. 17, No. 9, 2002, pp. 677683. doi:10.1046/j.1525-1497.2002.00746.X

[23] N. Crepaz, C. M. Lyles, R. J. Wolitski, W. F. Passin, S. M. Rama, J. H. Herbst, D. W. Purcell, R. M. Malow, R. Stall and HIV/AIDS Prevention Research Synthesis (PRS) Team, "Do Prevention Interventions Reduce HIV Risk Behaviors among People Living with HIV? A MetaAnalytic Review of Controlled Trials," AIDS, Vol. 20, No. 2, 2006, pp. 143-157. doi:10.1097/01.aids.0000196166.48518.a0

[24] G. K. Robbins, K. L. Johnson, Y. Chang, K. E. Jackson, P. E. Sax, J. B. Meigs and K. A. Freedberg, "Predicting Virologic Failure in an HIV Clinic," Clinical Infectious Diseases, Vol. 50, No. 5, 2010, pp. 779-786.

[25] P. Roux, M. P. Carrieri, V. Villes, P. Dellamonica, I. Poizot-Martin, I. Ravaux, B. Spire and MANIF2000 Cohort Study Group, "The Impact of Methadone or Buprenorphine Treatment and Ongoing Injection on Highly Active Antiretroviral Therapy (HAART) Adherence: Evidence from the MANIF2000 Cohort Study," Addiction, Vol. 103, No. 11, 2008, pp. 1828-1836. doi:10.1111/j.1360-0443.2008.02323.x 